\title{
Hypersensitivity to pyrazolones
}

\author{
Micha Levy
}

To the allergologist, hypersensitivity to drugs means immunological drug reactions as classified in the Gell and Coombs system. To others, it may also include non-dose related adverse drug reactions including the non-immune mediated idiosyncratic drug reactions (type B). In the following discussion the broad definition of idiosyncrasy including all type $\mathrm{B}$ reactions and a clinical approach rather than a mechanistic one will be used.

Pyrazolones are veteran drugs. Antipyrine was synthesised for clinical use in 1883. The methylated nitrogen derivative aminopyrine was introduced in 1897 and taken off the market in the 1970 s because of its propensity to form nitrosamines. Dipyrone has been in clinical use since 1922. In some countries it was banned because of the risk of agranulocytosis. In others it is the leading analgesic/antipyretic drug. Annual sales figures of pyrazolones amount to kilotons, mainly dipyrone and propyphenazone.

Unlike the acidic non-steroidal antiinflammatory drugs (NSAIDs) which are known to act on inflamed tissue by the inhibition of prostaglandin synthesis, pyrazolones produce analgesic/antipyretic effects associated with a much less potent anti-inflammatory effect on peripheral tissues. As shown in several animal species and also in humans, dipyrone acts on the central nervous system by the inhibition of prostanoids. ${ }^{2}$ Dipyrone has more than 20 known metabolites. ${ }^{3}$ A central hypothesis of immune mediated (hypersensitivity) drug reactions has been that drugs are metabolised to a reactive metabolite which can act as an hapten. ${ }^{4}$ There are limited reports concerning the immunogenic potential of pyrazolone and pyrazolidine metabolites. ${ }^{56}$ Genetic heterogeneity could also predispose patients to adverse drug reactions by the formation or inability to detoxify a reactive metabolite.

In 1977 Szczeklik et al put forward the hypothesis that, in sensitive patients, induction of asthmatic attacks by aspirin-like drugs is due to inhibition of tissue prostaglandin biosynthesis. In about $10 \%$ of adult asthmatics NSAIDS precipitate such attacks. ${ }^{8}$ Inhibition of cyclooxygenase leading to changes in the metabolism of arachidonic acid can also be the cause of urticaria/angio-oedema.

As for pyrazolones, hypersensitivity can be of two distinct forms. There are patients, usually with chronic asthma, whose idiosyncratic reactions resemble aspirin induced asthma and probably involve prostaglandin inhibition and overproduction of cysteinyl leukotrienes. The second group comprises patients who develop anaphylaxis, urticaria, and other forms of rash where the hypersensitivity seems to have an immunological background. ${ }^{9} 10$
Virtually all adverse reactions to pyrazolones are non-dose related. ${ }^{11}$ The following syndromes of pyrazolone hypersensitivity have been reported.

\section{Anaphylaxis}

Immediate hypersensitivity reactions are presumably IgE mediated and clinically characterised by laryngeal and angioneurotic oedema, generalised urticaria, bronchospasm, vasomotor collapse, and death. Patients with such reactions to pyrazolones display tolerance to NSAIDs. They often have a family history of allergic diseases.

Szczeklik ${ }^{12}$ described 22 patients who developed anaphylactic shock after taking a single dose of aminopyrine or dipyrone and another six patients in whom urticaria/angio-oedema occurred. All had positive skin tests to these drugs but oral challenge with NSAIDs was negative in all cases. There are no reliable in vitro tests for the diagnostic use of pyrazolone specific IgE. A history of pyrazolone hypersensitivity is the most important way to make the diagnosis. It has recently been suggested that a genetic predisposition to immediate hypersensitivity to pyrazolones is linked to the HLA DQ locus. ${ }^{13}$

Cases of anaphylaxis have been described following the use of dipyrone and amidopyrine ${ }^{1415}$ and occur following parenteral as well as oral administration. The estimation of risk of pyrazolone induced anaphylaxis, particularly following parenteral administration, by case-control studies is difficult because of the low exposure of control subjects and the unclear definition of the appropriate time of aetiologically significant exposure. In a study of a population of 14.5 million in Holland for two years an incidence of drug induced anaphylaxis of 3.7 per million annually was found. ${ }^{16}$ The excess mortality estimate associated with dipyrone use was 0.22 per 100 million.

Hoigne ${ }^{17}$ has described cases of severe hypotensive reaction following the intravenous administration of pyrazolones. The mechanism of these reactions is unclear. Kewitz et al reported that the risk of severe reactions induced by pyrazolone derivatives is no higher than that with opioids. ${ }^{18}$

\section{Cutaneous reactions}

Skin eruptions following aminopyrine and dipyrone include urticarial, morbilliform, scarlatiniform, erythematous, bullous, purpuric, exudative, and fixed lesions. ${ }^{19}$ The occurrence of IgE mediated hypersensitivity reactions to pyrazolones has been suggested by skin prick tests and intradermal testing. In other cases positive delayed responses to patch tests have been described. A case displaying both $\operatorname{IgE}$
Dr M Levy

mlevy@mdz.huji.ac.il 
mediated (type I) and a cell mediated (type IV) hypersensitivity reaction has recently been reported. ${ }^{20}$ In 1973 the Boston Collaborative Drug Surveillance Program noticed that drug rash occurred more frequently in Israel than in US patients. At that time $41.6 \%$ of Israeli patients received dipyrone, whereas in the USA dipyrone was not in use. The risk of dipyrone rash was estimated to be $2.4 \%$ of the exposed. However, it was recognised as being caused by the drug in only one third of the affected cases. ${ }^{21}$

In most cases dipyrone rash is mild. Rarely, it may also be part of a generalised drug reaction. Pyrazolone induced urticaria/angio-oedema can also be a manifestation of a pseudoallergic reaction, probably occurring via cyclooxygenase inhibition. ${ }^{810}$

Asero $^{22}$ has recently shown that three out of 34 patients $(9 \%)$ with a history of pyrazolone induced urticaria/angio-oedema had such reactions after aspirin administration. He recommended performing a simple single blind, placebo controlled drug challenge with aspirin in all patients with pyrazolone induced urticaria to prevent severe reactions in susceptible patients and to allow the safe use of NSAIDs in the others.

The more serious life threatening cutaneous complication is that of Stevens-Johnson's syndrome and toxic epidermal necrolysis (Lyell's syndrome). Cases have been reported following the use of pyrazolones and in combination with barbiturates. ${ }^{23}$ A large populationbased case-control study covering about 120 million inhabitants of France, Germany, Italy, and Portugal was conducted between the years 1989 and 1992 to quantify the risks associated with the use of specific drugs within the week preceding the first manifestation of the disease. Seven of the 245 cases $(3 \%)$ and $1 \%$ of the controls used pyrazolones. The association between both pyrazolones as a group and dipyrone and these conditions, according to the multivariate relative risk, is statistically not significant. $^{24}$

\section{Respiratory reactions}

Aspirin induced asthma has long been recognised and the diagnosis, symptomatology, and treatment of the syndrome have been described in detail. ${ }^{25}$ It is more common in patients with asthma, nasal polyps, rhinitis, and chronic urticaria. Current evidence indicates that cyclooxygenase inhibition and increased production of cysteinyl leukotriene plays an important part in these obstructive reactions. ${ }^{26-28}$ It has been shown that aspirin may cross react not only with certain NSAIDs, but also with pyrazolone derivatives including dipyrone ${ }^{929}$ in the precipitation of asthmatic attacks. The incidence and risk estimates of the asthma syndrome induced by pyrazolones are unknown.

\section{Agranulocytosis}

Agranulocytosis was first described by Schultz in $1922 .{ }^{30}$ In 1934 it was shown by rechallenge that amidopyrine was a cause of the disease. ${ }^{31}$ In the 1950 s evidence for the immunological pathogenesis of amidopyrine induced agranu- locytosis was provided by Moeschlin and Wagner. ${ }^{32}$ Since that time, however, conflicting data, judgements and regulations have prevailed as to the incidence of agranulocytosis and the use of pyrazolones in general, and dipyrone in particular. This has led to the conduct of one of the largest pharmacoepidemiological studies ever performed-The International Study of Agranulocytosis and Aplastic Anemia (IAAAS).$^{33}$ Its report on analgesics was published in $1986^{34}$ and a full description of the study experience appears as a monograph. ${ }^{35}$

Analgesic antipyretic drug use in the week before the clinical onset of illness - that is, the period of aetiological significance-was compared between 275 confirmed cases of agranulocytosis and 1636 hospital controls identified by the study centres in Jerusalem, Berlin, Ulm, Milan, Barcelona, Sofia, Budapest, and Stockholm. The study base comprised the total experience in these areas during the years 1980-6, which amounted to nearly 107 million person-years. There was significant regional variability in the rate ratio estimate for the use of dipyrone. In Ulm, Berlin, and Barcelona (grouped together) it was 23.7 (lower 95\% confidence interval (CI) 8.7), and for Israel it was 2.0 (lower CI 0.9). In Budapest and Sofia the estimates were close to unity, in Milan data were too sparse, while in Stockholm dipyrone was not in use. The excess risk estimate in Ulm, Berlin, and Barcelona connected with hospital admission for agranulocytosis from any dipyrone use in a seven day period amounted to 1.1 cases per million users.

The reason for the geographical variation in the risk of dipyrone induced agranulocytosis is intriguing. ${ }^{36}$ Efforts made by the investigators to detect the extent to which the variation is a reflection of methodological problems or hidden bias have not provided an answer. If real, regional differences could provide an important scientific lead in understanding the aetiology of the disease.

One hypothesis to explain causality in the order of magnitude of one case per million exposures is that several environmental and host factors act in concert. Viral infection could be such a factor. The rate ratio of patients with agranulocytosis having had a recent viral infection was estimated as 2.4 (95\% CI 1.5 to 3.9$)$. $^{37}$

The Swedish Medical Products Agency has recently reported that, since the reintroduction of dipyrone in August 1995, up to March 1999 seven cases of agranulocytosis were seen in patients who were reported to have used the drug. At the moment this can only be evaluated according to sales figures, which would translate to a much higher risk than that found by the IAAAS. Could this be an even more pronounced example of geographical variation in risk? Further assessment is required.

\section{Fever}

As for all drugs, the pyrazolone antipyretics can also cause drug fever. I have personally seen cases in which fever has resolved after dechal- 
lenge and appeared following the reintroduction of dipyrone. Similar cases have been reported by others. ${ }^{38}$

\section{Conclusions}

Mild forms of skin rash are relatively common. The incidence of pyrazolone induced asthma is unknown. Life threatening reactions, including Stevens-Johnson's syndrome, toxic epidermal necrolysis, anaphylaxis, and agranulocytosis are extremely rare. However, study and identification of subpopulations carrying higher risks (and, in particular, questions relating to the geographical variation of risk) and the acquisition of comparable quantitative data for all major adverse events to analgesic antipyretic drugs should be goals for the future.

1 Shimada SG, Otterness IG, Stitt JT. A study of the mechanism of action of the mild analgesic dipyrone. Agents Actions 1994:41:188-92.

2 Levy M, Brune K, Zylber-Katz E, et al. Cerebrospinal fluid prostaglandins after systemic dipyrone intake. Clin Pharmacol Ther 1998;64:107-12.

3 Levy M, Zylber-Katz E, Rosenkranz B. Kinetics of dipyrone and its metabolites. Clin Pharmacokinet 1995;28:216-34.

4 Uetrecht JP. Idiosyncratic drug reactions: possible role of reactive metabolites generated by leukocytes. Pharm Res $1989 ; 6: 265-73$

5 Schneider CH, Kasper MF, de Weck AL, et al. Diagnosis of antibody mediated drug allergy. Pyrazolone and pyrazolidinedione cross-reactivity relationship. Allergy 1987;42:597603.

6 Zhu D, Becker W, Schulz K, et al. Detection of IgE antibody specific for 1-phenyl-2,3 dimethyl-3-pyrazoline-one by RAST: a serological diagnostic method for sensitivity to pyrazoline drugs. Asian Pacific F Allergy Immunol 1992;10: 95-101.

7 Szczeklik A, Gryglewski RJ, Czerniawska-Mysik B. Clinical patterns of hypersensitivity to NSAID and their pathogenesis. 7 Allergy Clin Immunol 1977;60:276-84.

8 Szczeklik A. Adverse reactions to aspirin and non-steroidal anti-inflammatory drugs. Ann Allergy 1987;59:113-8.

Szczeklik A. Analgesics, allergy and asthma. Drugs 1986; 32 (Suppl 4):148 63 .

10 Czerniawska-Mysic G, Szczeklik A. Idiosyncrasy of pyrazolone drugs. Allergy 1981;36:3871-4.

11 Okonek S. Intoxication with pyrazolones. Br 7 Clin Pharma col 1980;10:385-9S

12 Szczeklik A. Analgesics, allergy and asthma. Br f Clin Pharmacol 1980;10:401-5s.

13 Kowalski ML, Woszcze G, Bienkiewicz B, et al. Association of pyrazolone drug hypersensitivity with HLA-DQ and DR antigen. Clin Exp Allergy 1998;28:1153-8.

14 Patriarca G, Venuti A, Bonini W. Allergy to pyramidon (aminopyrine) Ann Allergy 1973;31:84.

15 Lopatin AI, Luzhnyak AI, Surmina LM. Anaphylactic shock due to analgin. Klin Med (Mosk) 1972;50:115.

16 Van der Klauw MM, Stricker BHC, Herings RMC, et al. A population based case-cohort study of drug induced anaphylaxis. Br f Clin Pharmacol 1993;35:400-8.
17 Hoigne R, Stocker F, Barbier A, et al. Dual purpose of inpatient drug monitoring by physicians in two teaching hospitals in Berne. A. Patient adverse reaction surveillance in the hospital. Demonstration of the system by the detection of arterial hypotension due to novamine sulfone (Novalgin). In: Ducrot M, et al, eds. Computer aid to drug therapy and drug monitoring. Amsterdam: North Holland, 1978: 9-12.

18 Kewitz H, Harter G, Feldman U, et al. Observational cohort study in general practice: differences and equivalence

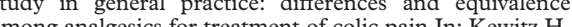
Roots I, Voight K, eds. Epidemiologic concepts in clinical pharmacology. Berlin: Springer Verlag, 1987: 73-86.

19 Voigtlander V. Adverse dermatological reactions to pyrazolones. Agents Actions Suppl 1986;19:303-11.

20 Bellegrandi S, Rosso R, Mattiacci G, et al. Combined immediate and delayed type hypersensitivity to Metamizol. Allergy 1999;54:88-90.

21 Levy $M$ for the Boston Collaborative Drug Surveillance Program. Dipyrone as a cause of drug rashes. Int $\mathcal{F}$ Epidemiol 1973;2:167-70.

22 Asero R. Detection of aspirin reactivity in patients with pyrazolone-induced disorders. Allergy 1998;53:214-5.

23 Schulz KH, Glowania HJ. Lyell syndrome. Allergologie 1983;6:450-3.

24 Roujeau JC, Kelly JP, Naldi L, et al. Medication use and the risk of Steven-Johnson syndrome or toxic epidermal necrolysis. N Engl f Med 1995;33:1600-7.

25 Virchow C, Schmitz-Schumann M, Juhl-Schaub E. Pyrazolones and analgesic asthma syndrome. Agens Action Suppl 1986;19:291-301.

26 Israel E, Fischer AR, Rosenberg MA, et al. The pivotal role of 5-lipoxygenase products in the reaction of aspirinsensitive asthmatics to aspirin. Am Rev Respir Dis 1993; 148: 1447-51.

27 Sampson AP. The leukotrienes: mediators of chronic inflammation in asthma. Clin Exp Allergy 1996;261:9951004

28 Yoshida S, Nakagawa H, Yamawaki Y, et al. Bronchial hyperresponsiveness, hypersensitivity to analgesics and urinary leukotriene excretion in patients with aspirin intolerant asthma. Int Arch Allergy Irnmunol 1998;117:146-51.

29 Bartoli E, Faedda-in-Masala R, Chiandussi L. Druginduced asthma (letter). Lancet 1976;i:1357.

30 Schultz W. Uber eigenartige Halserkrankungen. Dtsch Med Wschr 1922;48:1495.

31 Madison FW, Squier TL. The etiology of primary granulocytopenia. $\mathcal{F A M A}$ 1934;102:755.

32 Moeschlin S, Wagner K. Agranulocytosis due to the occurrence of leukocyte agglutinins. Acta Haematol 1952;8:29.

33 International Agranulocytosis and Aplastic Anemia Study. The design of a study of the drug etiology of agranulocytosis and aplastic anemia. Eur f Clin Pharmacol 1983;24:83366 .

34 International Agranulocytosis and Aplastic Anemia Study. Risks of agranulocytosis and aplastic anemia. A first report of their relation to drug use with special reference to analgesics. ҰAMA 1986;256:1749-57.

35 Kaufman DW, Kelly JP, Levy M, et al. The drug etiology of agranulocytosis and aplastic anemia. Oxford: Oxford University Press, 1991

36 Faich GFA. Analgesic risk and pharmacoepidemiology. ҰAMA 1986;256:178.

37 Levy $M$. Role of viral infections in the induction of advers drug reactions. Drug Safety 1997;16:1-8.

38 Guerrero M, Basomba A, Garcia-Villalmanzo I, et al. Druginduced fever caused by pyrazolones (letter). Med Clin Barc 1984;82:868. 